Mathematical Research Letters 1, 797-808 (1994)

\title{
THE GENUS OF EMBEDDED SURFACES IN THE PROJECTIVE PLANE
}

\author{
P. B. Kronheimer and T. S. Mrowka \\ A в sтRAст. We show how the new invariants of 4-manifolds resulting from \\ the Seiberg-Witten monopole equation lead quickly to a proof of the 'Thom \\ conjecture'.
}

\section{Statement of the result}

The genus of a smooth algebraic curve of degree $d$ in $\mathbb{C P}^{2}$ is given by the formula $g=(d-1)(d-2) / 2$. A conjecture sometimes attributed to Thom states that the genus of the algebraic curve is a lower bound for the genus of any smooth 2-manifold representing the same homology class. The conjecture has previously been proved for $d \leq 4$ and for $d=6$, and less sharp lower bounds for the genus are known for all degrees $[5,6,7,10,14]$. In this note we confirm the conjecture.

Theorem 1. Let $\Sigma$ be an oriented 2-manifold smoothly embedded in $\mathbb{C P}^{2}$ so as to represent the same homology class as an algebraic curve of degree $d$. Then the genus $g$ of $\Sigma$ satisfies $g \geq(d-1)(d-2) / 2$.

Very recently, Seiberg and Witten $[12,13,15]$ introduced new invariants of 4-manifolds, closely related to Donaldson's polynomial invariants [2], but in many respects much simpler to work with. The new techniques have led to more elementary proofs of many theorems in the area. Given the monopole equation and the vanishing theorem which holds when the scalar curvature is positive (something which was pointed out by Witten), the rest of the argument presented here is not hard to come by. A slightly different proof of the Theorem, based on the same techniques, has been found by Morgan, Szabo and Taubes.

1991 Mathematics Subject Classification. Primary 57R57, 57N13, 57R55; Secondary 57R40, 14J60.

Received November 9, 1994.

First author supported by an Advanced Fellowship from the EPSRC. Second author partially supported by NSF/NYI grant number DMS-9357641 and a Sloan Fellowship. 
It is also possible to prove a version of Theorem 1 for other complex surfaces, without much additional work, so reproducing and extending results of [8] and [9]. This and various other applications will be treated in a later paper, with joint authors.

\section{The monopole equation and the Seiberg-Witten invariants}

Let $X$ be an oriented, closed Riemannian 4-manifold. Let a $\operatorname{spin}^{c}$ structure on $X$ be given. We write $c$ for the $\operatorname{spin}^{c}$ structure and write $W^{+}=W_{c}^{+}$ and $W^{-}=W_{c}^{-}$for the associated $\operatorname{spin}^{c}$ bundles. Thus $W^{+}$is a $U(2)$ bundle and Clifford multiplication leads to a linear isomorphism $\rho=\rho_{c}$ from $\Lambda^{+}(X) \otimes \mathbb{C}$ to the associated $P S L(2, \mathbb{C})$ bundle:

$$
\rho: \Lambda^{+} \otimes \mathbb{C} \rightarrow \mathfrak{s l}\left(W^{+}\right) .
$$

The usual conventions ensure that $\rho$ carries the real forms to $\mathfrak{s u}\left(W^{+}\right)$and that if $e_{i}(i=1, \ldots, 4)$ are an oriented orthonormal frame at a point then $\rho\left(e_{1} \wedge e_{2}+e_{3} \wedge e_{4}\right)$ has eigenvalues $\pm 2 i$. There is a pairing

$$
\sigma: W^{+} \times \bar{W}^{+} \rightarrow \mathfrak{s l}\left(W^{+}\right)
$$

modelled on the map $\mathbb{C}^{2} \times \overline{\mathbb{C}}^{2} \rightarrow \mathfrak{s l}(2)$ given by

$$
(v, w) \mapsto i\left(v \bar{w}^{t}\right)_{o},
$$

where the subscript means the trace-free part. Let $L$ denote the determinant of $W^{+}$, a line bundle on $X$. The equations introduced by Seiberg and Witten are the following pair of equations for a unitary connection $A$ in $L$ and a section $\Phi$ of $W^{+}$:

$$
\begin{aligned}
D_{A} \Phi & =0 \\
\rho\left(F_{A}^{+}\right) & =i \sigma(\Phi, \Phi) .
\end{aligned}
$$

Here $D_{A}: \Gamma\left(W^{+}\right) \rightarrow \Gamma\left(W^{-}\right)$is the Dirac operator on the spin ${ }^{c}$ bundle, and $F_{A}^{+}$is the self-dual part of the curvature of $A$. The gauge group $\operatorname{Map}\left(X, S^{1}\right)$ acts on the set of solutions via its action as scalar automorphisms of $W^{+}$, and we write $M_{c}$ for the moduli space, the quotient of the set of solutions by the gauge group. We call a solution irreducible if $\Phi$ is not identically zero. Such solutions form free orbits of the gauge group, while the reducible solutions $(\Phi=0)$ have stabilizer $S^{1}$.

If the homology of $X$ has no 2-torsion, the $\operatorname{spin}^{c}$ structure $c$ is entirely determined by the topological type of $L$, which may be any line bundle whose first Chern class is an integral lift of $w_{2}$. In general, when $L$ is fixed, the $\operatorname{spin}^{c}$ structures with determinant $L$ are a principal space for the 2torsion part of $H^{2}(X ; \mathbb{Z})$. When necessary, we write $M_{c}(g)$ to indicate the dependence on the Riemannian metric $g$. 
Lemma 2. Any solution $(A, \Phi)$ satisfies the $C^{0}$ bound

$$
|\Phi|^{2} \leq \max (0,-s)
$$

at the points where $|\Phi|$ is maximum. Here $s$ is the scalar curvature of $X$.

Proof. The Weitzenböck formula for the Dirac operator reads

$$
D_{A}^{*} D_{A} \Phi=\nabla_{A}^{*} \nabla_{A} \Phi+\frac{s}{4} \Phi-\frac{1}{2} \rho\left(F_{A}^{+}\right) \Phi .
$$

At points where $|\Phi|^{2}$ is maximum we calculate

$$
\begin{aligned}
0 & \leq \Delta|\Phi|^{2} \\
& =2\left\langle\nabla_{A}^{*} \nabla_{A} \Phi, \Phi\right\rangle-2\left\langle\nabla_{A} \Phi, \nabla_{A} \Phi\right\rangle \\
& \leq 2\left\langle\nabla_{A}^{*} \nabla_{A} \Phi, \Phi\right\rangle \\
& =-\frac{s}{2}|\Phi|^{2}+\langle i \sigma(\Phi, \Phi) \Phi, \Phi\rangle \\
& =-\frac{s}{2}|\Phi|^{2}-\frac{1}{2}|\Phi|^{4} .
\end{aligned}
$$

The identity used in the last line can be verified from the model of $\sigma$ given above. If $|\Phi|^{2}$ is non-zero at the maximum, we may divide by $|\Phi|^{2}$ to obtain the bound $|\Phi|^{2} \leq-s$.

Corollary 3. If $\left(A_{i}, \Phi_{i}\right)$ is a sequence of solutions on $X$, then there is a subsequence $\left\{i^{\prime}\right\} \subset\{i\}$ and gauge transformations $g_{i^{\prime}}$ such that the sequence $g_{i^{\prime}}\left(A_{i^{\prime}}, \Phi_{i^{\prime}}\right)$ converges in $C^{\infty}$. Thus $M_{c}$ is compact.

Proof. The $C^{0}$ bound on $\Phi$ makes this entirely straightforward. Choose any smooth connection $B$ in $L$. We can find gauge transformations $g_{i}^{o}$ in the identity component of $\operatorname{Map}\left(X, S^{1}\right)$ so that the 1 -form $g_{i}^{o}\left(A_{i}\right)-B$ is coclosed. The component group of $\operatorname{Map}\left(X, S^{1}\right)$ is isomorphic to $H^{1}(X ; \mathbb{Z})$, and by choosing a gauge transformation $g_{i}$ from an appropriate component, we can arrange further that the harmonic part of $g_{i}\left(A_{i}\right)-B$ is bounded, independent of $i$, because $H^{1}(X ; \mathbb{R}) / H^{1}(X ; \mathbb{Z})$ is compact. The equations and the $C^{0}$ bound on $\Phi$ then give an $L_{1}^{p}$ bound on $g_{i}\left(A_{i}\right)-B$ as well as on $g_{i}\left(\Phi_{i}\right)$ for any $p$. By a bootstrapping argument, one obtains bounds on all higher derivatives (depending on $B$ ). There is therefore a convergent subsequence.

On a manifold with boundary, a suitable gauge-fixing condition for a 1-form $g^{o}(A)-B$ is that it be co-closed and annihilate the normal vectors at the boundary. The argument above then proves: 
Lemma 4. If $Z$ is a compact, oriented Riemannian 4-manifold with boundary, equipped with a spin ${ }^{c}$ structure, and if $\left(A_{i}, \Phi_{i}\right)$ is a sequence of solutions on $Z$ with $\left|\Phi_{i}\right|$ uniformly bounded, then there is a subsequence $\left\{i^{\prime}\right\} \subset\{i\}$ and gauge transformations $g_{i^{\prime}}$ such that the sequence $g_{i^{\prime}}\left(A_{i^{\prime}}, \Phi_{i^{\prime}}\right)$ converges in $C^{\infty}$.

The monopole equations $(*)$ can be perturbed in a gauge-invariant fashion by adding a form to the right-hand side of the second equation: if $\delta \in \Omega^{+}(X)$, we consider the equations

$$
\begin{aligned}
D_{A} \Phi & =0 \\
\rho\left(F_{A}^{+}+i \delta\right) & =i \sigma(\Phi, \Phi) .
\end{aligned}
$$

Let $M_{c, \delta}$ denote the moduli space of solutions. A $C^{0}$ bound on $|\Phi|^{2}$ can be obtained from the equations as before (one only has to replace $-s$ in Lemma 2 by $-s+2|\rho(\delta)|)$. Let $\mathcal{M}_{c}$ denote the parametrized moduli space, that is:

$$
\begin{aligned}
\mathcal{M}_{c} & =\bigcup_{\delta \in \Omega^{+}} M_{c, \delta} \times\{\delta\} \\
& \subset\left(\mathcal{A} \times \Gamma\left(W^{+}\right) \times \Omega^{+}\right) / \operatorname{Map}\left(X, S^{1}\right),
\end{aligned}
$$

where $\mathcal{A}$ is the space of unitary connections in $L$. It is also convenient to allow $A$ and $\Phi$ to be of class $L_{k}^{2}$, the form $\delta$ to be of class $L_{k-1}^{2}$ and the gauge transformations to be of class $L_{k+1}^{2}$ for some large $k$, so that $\mathcal{M}_{c}^{*}$ is a subset of a Banach manifold. Here we are writing $M_{c}^{*}$ and $\mathcal{M}_{c}^{*}$ for the moduli space and parametrized moduli space of irreducible solutions. We omit mention of $k$ from our notation.

Lemma 5. The irreducible part of the parametrized moduli space $\mathcal{M}_{c}^{*}$ is a smooth manifold. The projection $\pi: \mathcal{M}_{c}^{*} \rightarrow \Omega^{+}$is Fredholm of index

$$
d(L)=\frac{1}{4}\left(c_{1}(L)^{2}-(2 \chi+3 \sigma)\right),
$$

where $\chi$ and $\sigma$ are the Euler number and signature of $X$. The projection of the whole space $\mathcal{M}_{c}$ to $\Omega^{+}$is proper.

Proof. Let $(A, \Phi, \delta)$ be a solution of the equations with $\Phi$ non-zero. The linearization of the equations at this point is the operator

$$
P: \Omega^{1}(i \mathbb{R}) \times \Gamma\left(W^{+}\right) \times \Omega^{+} \rightarrow \Gamma\left(W^{-}\right) \times i \mathfrak{s u}\left(W^{+}\right)
$$

given by

$$
P(a, \phi, \epsilon)=\left(D_{A} \phi+a \Phi, \rho\left(d^{+} a+i \epsilon\right)+2 \operatorname{Im} \sigma(\phi, \Phi)\right) .
$$


To establish surjectivity, it is sufficient to show that no element $(\psi, \kappa)$ in the range can be $L^{2}$-orthogonal to the image of $P$. But if $(\psi, \kappa)$ is orthogonal, then by varying $\epsilon$ alone one can first see that $\kappa$ is zero. Then by varying $a$ alone and using the fact that $\Phi$, being a solution of the Dirac equation, cannot vanish on an open set, one sees that $\psi$ is zero also. So the set of irreducible solutions is a manifold, by the implicit function theorem. The gauge group acts freely, and the quotient $\mathcal{M}_{c}^{*}$ is a manifold also, as slices for the gauge group action can be constructed using the Coulomb gauge fixing condition.

To calculate the Fredholm index taking account of the Coulomb condition, one must calculate the index of a linear operator $P+d^{*}$ which is a compact perturbation of the sum of the two operators

$$
\begin{gathered}
D_{A}: \Gamma\left(W^{+}\right) \rightarrow \Gamma\left(W^{-}\right) \\
d^{*}+d^{+}: \Omega^{1} \rightarrow \Omega^{0} \oplus \Omega^{+} .
\end{gathered}
$$

The real index of the first is $\frac{1}{4} c_{1}(L)^{2}-\frac{1}{4} \sigma$, and the index of the second is $-\frac{1}{2}(\chi+\sigma)$. The sum of these two indices is $d(L)$. The last clause of the Lemma is, like Corollary 3, a consequence of Lemma 2.

We can now define a simple mod 2 version of the invariants of Seiberg and Witten. Suppose we have a $\operatorname{spin}^{c}$ structure $c$ such that the determinant bundle $L$ satisfies $d(L)=0$. (Such a $\operatorname{spin}^{c}$ structure may or may not exist: the condition is the same as that for $X$ to have an almost complex structure). Call a Riemannian metric $g$ 'good' if the image of $c_{1}(L)$ in real cohomology is not represented by an anti-self-dual form. If $g$ is good, then there exists a positive $\epsilon$ such that the equation $F_{A}^{+}+i \delta=0$ has no solutions when $|\delta|<\epsilon$ (we may take the $C^{0}$ norm). Let $\delta$ be a regular point of the projection $\pi$ in the Lemma above with $|\delta|<\epsilon$, so that the fibre $M_{c, \delta}^{*}=M_{c, \delta}$ is a compact zero-manifold. Define $n_{c}(g)$ to be the number of points in this moduli space, counted mod 2:

$$
n_{c}(g)=\# M_{c, \delta}(g) \quad(\bmod 2) .
$$

If $\delta_{1}$ and $\delta_{2}$ are both regular values with $C^{0}$ norm less than $\epsilon$, we can join them by a path which is transverse to $\pi$; the inverse image of this path is a compact 1-manifold, with boundary the union of the two moduli spaces, so $n_{c}(g)$ is independent of $\delta$. Similarly, if $g_{1}$ and $g_{2}$ are two good metrics which can be joined by a path of good metrics, then $n_{c}\left(g_{1}\right)=n_{c}\left(g_{2}\right)$.

As shown in [1], if $b^{+}$is bigger than 1 and $L$ is non-trivial, the space of good metrics is path-connected. In this case, $n_{c}$ is an invariant of the pair $(X, c)$ alone, independent of the metric. Our application of the invariants, however, deals with a manifold with $b^{+}=1$. 


\section{The invariants of $\mathbb{C P}^{2} \# n \overline{C P}^{2}$}

Let $\mathbb{C P}^{2}$ be the complex projective plane with its standard orientation, and let $X$ be the manifold $\mathbb{C P}^{2} \# n \overline{C P}^{2}$. As a basis for the second cohomology, we take in $\mathbb{C P}^{2}$ the Poincaré dual $H$ of the class represented by the projective line with its complex orientation, and in the $i$ th copy of $\overline{C P}^{2}$ a similar class which we call $E_{i}$. The quadratic form on $H^{2}(X ; \mathbb{R})$ has signature $(1, n)$, and we write $C^{+}$for the component of the open positive cone in $H^{2}(X ; \mathbb{R})$ which contains $H$. For each Riemannian metric on $g$, there is a self-dual harmonic form $\omega_{g}$ whose cohomology class lies in $C^{+}$. This form is unique up to positive scaling. The condition that a metric $g$ be good can be rephrased as the condition $c_{1}(L) \smile\left[\omega_{g}\right] \neq 0$.

The manifold $X$ is a complex manifold in a standard way, as the blowup of the projective plane at $n$ points. There is therefore a preferred $\operatorname{spin}^{c}$ structure $c$. The corresponding line bundle $L$ has $c_{1}(L)=c_{1}(X)$ and $d(L)=0$. In terms of the basis introduced above, and writing $E=\sum E_{i}$, we have

$$
c_{1}(L)=3 H-E .
$$

Note that since $\left[\omega_{g}\right]$ is a class of positive square, we cannot have $c_{1}(L) \smile$ $\left[\omega_{g}\right]=0$ unless $c_{1}(L)$ has negative square; that is, unless $n>9$.

Proposition 6. For this standard spinc structure, the invariant $n_{c}(g)$ for a good metric $g$ is $0 \bmod 2$ if $c_{1}(L) \smile\left[\omega_{g}\right]$ is positive, and $1 \bmod 2$ if $c_{1}(L) \smile\left[\omega_{g}\right]$ is negative.

Proof. Let $g_{t}(t \in[-1,1])$ be a smooth path of Riemannian metrics whose endpoints are good. By the argument of [1], we can perturb the path so that the path $\left[\omega_{g_{t}}\right]$ in $C^{+}$is transverse to the hyperplane $c_{1}(L)^{\perp}$. Let $k$ be the number of times $\left[\omega_{g_{t}}\right]$ meets the hyperplane (which is the number of times that $c_{1}(L) \smile\left[\omega_{g_{t}}\right]$ changes sign). We first show that $n_{c}\left(g_{-1}\right)$ and $n_{c}\left(g_{1}\right)$ differ by the parity of $k$.

Without loss of generality, we assume that $c_{1}(L) \smile\left[\omega_{g_{t}}\right]$ changes sign just once, at $t=0$. The parametrized moduli space

$$
\mathbf{M}_{c}=\bigcup_{t} M_{c}\left(g_{t}\right) \times\{t\}
$$

then contains a single reducible point, in the fibre over 0 . Choose a $\delta \in \Omega^{+}$ so as to perturb the equations, and make it sufficiently small that the expression

$$
f(t)=c_{1}(L) \smile\left[\omega_{g_{t}}\right]+2 \pi \int \delta \wedge \omega_{g_{t}}
$$


changes sign once, transversely. We may assume this happens still at $t=0$. There is then a single reducible solution of the perturbed equations at $t=0$, and at all other points the perturbed space $\mathbf{M}_{c, \delta}$ is a smooth 1-manifold, with boundary the union of $M_{c, \delta}\left(g_{1}\right)$ and $M_{c, \delta}\left(g_{-1}\right)$. We must show that an odd number of arcs emerge from the reducible point.

Let us examine the Kuranishi model of $\mathbf{M}_{c, \delta}$ in the neighbourhood of the reducible solution $\left(A_{0}, 0\right)$. The model is $\psi^{-1}(0) / S^{1}$, where $\psi$ is (the germ of) an $S^{1}$-equivariant map

$$
\psi: \mathbb{R} \oplus \operatorname{Ker}\left(D_{A_{0}}\right) \rightarrow \operatorname{Coker}\left(d^{+}\right) \oplus \operatorname{Coker}\left(D_{A_{0}}\right)
$$

and the $S^{1}$ action is the standard scalar multiplication on the kernel and cokernel of $D_{A_{0}}$. The $\mathbb{R}$ on the left-hand side represents the $t$ coordinate. On the right-hand side, $\operatorname{Coker}\left(d^{+}\right)$is 1-dimensional, and if $\operatorname{Coker}\left(D_{A_{0}}\right)$ has complex dimension $r$ then $\operatorname{Ker}\left(D_{A_{0}}\right)$ has dimension $r+1$ by the index theorem. If we identify $\operatorname{Coker}\left(d^{+}\right)$with $\mathbb{R}$ via the map $\theta \mapsto \int \theta \wedge \omega_{g_{0}}$, then we can calculate

$$
\left.\frac{\partial \psi}{\partial t}\right|_{(0,0)}=\frac{i}{2 \pi}\left(f^{\prime}(0), 0\right),
$$

following [1]. We have arranged that $f^{\prime}(0)$ is non-zero, so the local model can be reduced to $\psi_{1}^{-1}(0) / S^{1}$, for some $\psi_{1}: \mathbb{C}^{r+1} \rightarrow \mathbb{C}^{r}$. Removing the singular point, we obtain a model for a deleted neighbourhood of the reducible in $\mathbf{M}_{c, \delta}$ as the zero set of a section $\bar{\psi}_{1}$ of the bundle $\mathbb{C}^{r} \otimes \mathcal{O}(1)$ on $\mathbb{C P}^{r} \times \mathbb{R}^{+}$. Here $\mathcal{O}(1)$ is the line bundle of degree 1 on $\mathbb{C P}^{r}$, pulled back to the punctured cone

$$
\mathbb{C P}^{r} \times \mathbb{R}^{+}=\left(\mathbb{C}^{r+1} \backslash\{0\}\right) / S^{1}
$$

The zero set of $\bar{\psi}_{1}$ is transverse, by our assumption on the smoothness of $\mathbf{M}_{c, \delta}^{*}$. For an open dense set of numbers $\epsilon, \bar{\psi}_{1}^{-1}(0)$ meets $\mathbb{C P}^{r} \times\{\epsilon\}$ in an odd number of points, because the Euler number of the bundle $\mathbb{C}^{r} \otimes \mathcal{O}(1)$ on $\mathbb{C P}^{r}$ is 1 . This completes the proof that $n_{c}\left(g_{-1}\right)$ and $n_{c}\left(g_{1}\right)$ differ in parity as claimed.

To complete the proof of Proposition 6, it is sufficient to exhibit a metric $g_{+}$for which $c_{1}(L) \smile\left[\omega_{g_{+}}\right]$is positive and for which $M_{c}\left(g_{+}\right)$is empty. In [4] it is shown that $\mathbb{C P}^{2} \# n \overline{C P}^{2}$ admits Kähler metrics $g_{+}$of positive scalar curvature. In the Kähler situation, $\omega_{g}$ is a positive multiple of the Kähler form, and its cup-product with $c_{1}(L)$ has the same sign as the scalar curvature. The moduli space for this metric is empty by Lemma 2, so both of our requirements are satisfied. An alternative way to obtain a metric $g_{+}$ with the required properties is to use the constructions of [11] and [3].

Corollary 7. If $g$ is a Riemannian metric on $X=\mathbb{C P}^{2} \# n \overline{\mathbb{C P}}^{2}$ with $c_{1}(L) \smile\left[\omega_{g}\right]$ negative, then the moduli space $M_{c}(g)$ for the line bundle $L$ with $c_{1}(L)=c_{1}(X)$ is non-empty. 


\section{Solutions on tubes}

We return to a general oriented Riemannian 4-manifold having a $\operatorname{spin}^{c}$ structure $c$ of determinant $L$. Suppose $X$ contains an oriented 3-manifold $Y$ which separates $X$ into two components $X_{-}$and $X_{+}$with common boundary, and suppose that the Riemannian metric is a product $[-\epsilon, \epsilon] \times Y$ in a collar of $Y$. Let $\left(X_{R}, g_{R}\right)$ be the Riemannian manifold obtained from $X$ by cutting open along $Y$ and inserting a cylinder $[-R, R] \times Y$. (So $g_{0}$ is the original metric.) We identify $X_{R}$ with $X$ and write $M_{c}\left(g_{R}\right)$ for the moduli space.

Proposition 8. Suppose the moduli space $M_{c}\left(g_{R}\right)$ is non-empty for all sufficiently large $R$. Then there exists a solution of the equations on the cylinder $\mathbb{R} \times Y$ which is translation-invariant in a temporal gauge. (The spin $^{c}$ structure on the cylinder is the restriction of the spin ${ }^{c}$ structure on $X$.

(After identifying the line bundle $L$ on $\mathbb{R} \times Y$ with the pull-back of a line bundle on $Y$, we say that a solution $(A, \Phi)$ is in temporal gauge if the $d t$ component of $A$ vanishes.)

Proof. On the cylinder $\mathbb{R} \times Y$, the two $\operatorname{spin}^{c}$ bundles $W^{+}$and $W^{-}$can be identified using Clifford multiplication by $d t$, and both can be identified with the pull-back of the $U(2)$ bundle $W_{3} \rightarrow Y$ associated to the $\operatorname{spin}^{c}$ structure which $Y$ obtains. Let $A(t)$ be the path of connections in $L \rightarrow Y$ obtained by restricting $A$ to the slices $\{t\} \times Y$, and let $\Phi(t)$ be the path in $\Gamma\left(W_{3}\right)$ obtained similarly via the above identifications. Let $\partial_{A}: \Gamma\left(W_{3}\right) \rightarrow$ $\Gamma\left(W_{3}\right)$ be the self-adjoint Dirac operator on the 3 -manifold. If $A$ is in temporal gauge, it can be recovered from the path $A(t)$ in the space $\mathcal{A}_{3}$ of connections on $Y$.

In temporal gauge, the equations $(*)$ become the following equations for $A(t)$ and $\Phi(t)$ :

$$
\begin{aligned}
& d A / d t=-* F_{A}+i \tau(\Phi, \Phi) \\
& d \Phi / d t=-\partial_{A} \Phi .
\end{aligned}
$$

Here $\tau$ is a pairing from $W_{3} \times \bar{W}_{3}$ to $\Lambda^{1}(Y)$, obtained from Clifford multiplication by using the hermitian metric on $W_{3}$. These are the downward gradient flow equations for the functional $\frac{1}{2} C$ on $\mathcal{A}_{3} \times \Gamma\left(W_{3}\right)$ given by

$$
C(A, \Phi)=\int(A-B) \wedge F_{A}+\int\left\langle\Phi, \partial_{A} \Phi\right\rangle .
$$

Here $B$ is any chosen connection on $L \rightarrow Y$, made necessary by the fact that $\mathcal{A}_{3}$ has no preferred base-point in general. 
This function is invariant under gauge transformations $h$ belonging to the identity component of the gauge group $\operatorname{Map}\left(Y, S^{1}\right)$, but if $h$ has a non-trivial class $[h] \in H^{1}(X ; \mathbb{Z})$ then

$$
C(h(A), h(\Phi))=C(A, \Phi)+8 \pi^{2} c_{1}(L) \smile[h] .
$$

Let $\gamma$ be a 1-dimensional cycle in $Y$ dual to $c_{1}(L)$. As $Y$ sits in $X$, this cycle is the intersection with $Y$ of a 2-cycle $\Gamma$ in $X$ dual to $c_{1}(L)$ on $X$. Thus $\gamma$ bounds relative cycles $\Gamma_{+}$and $\Gamma_{-}$in $X_{+}$and $X_{-}$.

Now suppose that $\left(A_{R}, \Phi_{R}\right)$ is a solution of the equations on $\left(X_{R}, g_{R}\right)$, defined for all sufficiently large $R$. Take a reference connection $B_{R}$ on $L \rightarrow$ $X_{R}$ obtained from fixed connections $B_{ \pm}$over the pieces $X_{ \pm}$(independent of $R$ ) and a connection $[-R, R] \times B$ on the cylinder (after again choosing an identification of $L$ on the cylinder with the pull-back of a bundle on $Y$ ). Without loss of generality, $A_{R}$ is in temporal gauge with respect to the chosen product structure over the cylinder.

Let $l(R)$ be the change in $C$ along the cylinder:

$$
l(R)=C\left(A_{R}(R), \Phi_{R}(R)\right)-C\left(A_{R}(-R), \Phi_{R}(-R)\right) .
$$

This quantity is negative and independent of all choices. We claim that $l(R)$ satisfies a uniform bound, independent of $R$. To see this, note first that the argument of Corollary 3 and Lemma 4 means that we can choose gauge transformations $h_{R}^{+}$and $h_{R}^{-}$over $X_{+}$and $X_{-}$so that $h_{R}^{ \pm}\left(A_{R}\right)-B_{ \pm}$ and its first derivatives are uniformly bounded. We therefore have uniform bounds on each of the two quantities

$$
C\left(h_{R}^{ \pm}\left(A_{R}\right)( \pm R), h_{R}^{ \pm}\left(\Phi_{R}\right)( \pm R)\right) .
$$

However, since $\gamma$ is the boundary of a chain $\Gamma_{ \pm}$in $X_{ \pm}$, the degree of $h_{R}^{ \pm}$ on $\gamma \subset Y$ is zero. From the formula above for how $C$ changes under gauge transformation, it follows that

$$
C\left(h_{R}^{ \pm}\left(A_{R}\right)( \pm R), h_{R}^{ \pm}\left(\Phi_{R}\right)( \pm R)\right)=C\left(A_{R}( \pm R), \Phi_{R}( \pm R)\right) .
$$

This gives the desired bound on $l(R)$.

Since the equations are of a gradient flow, the value of $C$ changes monotonically along the cylinder. Taking $R$ to be an integer $N$, we see that there must be, for each $N$, a solution of the equations on $[0,1] \times Y$ for which the change in $C$ along the interval is at most $1 / N$. Furthermore, since the infimum of the scalar curvature of $X_{R}$ is independent of $R$, these solutions satisfy a uniform $C^{0}$ bound, by Lemma 2. Taking a sequence of such solutions with $N$ tending to $\infty$, we deduce from Lemma 4 that there is a solution on $[0,1] \times Y$ for which $C$ is constant. Such a solution is translation-invariant in a temporal gauge, and can be extended to a translation-invariant solution on $\mathbb{R} \times Y$. 


\section{A vanishing argument}

We continue to consider a cylinder $\mathbb{R} \times Y$, but we now take $Y$ to be a Riemannian product $S^{1} \times \Sigma$, where $\Sigma$ is a 2-manifold of constant scalar curvature. We choose to make $\Sigma$ of unit area, so that its scalar curvature is $-2 \pi(4 g-4)$. (The scalar curvature is twice the Gaussian curvature). We assume the genus $g$ is at least 1 .

Lemma 9. If there exists a solution to the equations $(*)$ on $\mathbb{R} \times Y$ which is translation-invariant in a temporal gauge, then $c_{1}(L)[\Sigma]$ is not greater than $2 g-2$ in absolute value.

Proof. Suppose that $(A, \Phi)$ is a solution which is translation-invariant in a temporal gauge. Because $Y$ is compact, $|\Phi|$ achieves its supremum, so from Lemma 2 we obtain the $C^{0}$ bound $|\Phi|^{2} \leq 2 \pi(4 g-4)$. This translates into the bound $|\sigma(\Phi, \Phi)| \leq \sqrt{2} \pi(4 g-4)$, where the norm on $\mathfrak{s u}\left(W^{+}\right)$is the one coming from the inner product $\operatorname{tr}\left(a^{*} b\right)$ (see the model for $\sigma$ in section 2.) From the first equation of $(*)$ we then obtain

$$
\left|F_{A}^{+}\right| \leq \sqrt{2} \pi(2 g-2)
$$

because $\rho$ increases lengths by a factor of 2 . Because $A$ is translationinvariant in a temporal gauge, the curvature $F_{A}$ is a 2-form pulled back from $Y$. From this it follows that $F_{A}^{+}$and $F_{A}^{-}$have the same magnitude, and we therefore have

$$
\left|F_{A}\right| \leq 2 \pi(2 g-2)
$$

and hence

$$
\begin{aligned}
\left|c_{1}(L)[\Sigma]\right| & =\left|\frac{i}{2 \pi} \int_{\Sigma} F_{A}\right| \\
& \leq \frac{1}{2 \pi} \sup \left|F_{A}\right| \operatorname{Area}(\Sigma) \\
& \leq 2 g-2 .
\end{aligned}
$$

\section{Proof of the Theorem}

We now turn to the proof of Theorem 1 . Let $\Sigma$ be a smoothly embedded oriented surface, dual to the class $d H$ in $\mathbb{C P}^{2}$. If $d$ is 1 or 2 , the inequality of the Theorem is trivial, while if $d$ is 3 the result was known to Kervaire and Milnor [5]. We therefore assume that $d$ is greater than 3. In this range we may suppose, without loss of generality, that $\Sigma$ is not a sphere.

Regard $\Sigma$ now as living in the connected sum $X=\mathbb{C P}^{2} \# d^{2} \overline{C P}^{2}$. Let $S_{i}$ be a sphere in the $i$ th copy of $\overline{\mathbb{C P}}^{2}$, dual to the class $-E$, and let $\tilde{\Sigma} \subset X$ be 
the surface formed by taking an internal connected sum of $\Sigma$ with all the $S_{i}$. This surface is dual to the class $d H-E$, where $E=\sum E_{i}$ as before. It has the same genus as $\Sigma$ and has trivial normal bundle. We take the canonical $\operatorname{spin}^{c}$ structure $c$ on $X$, so that $c_{1}(L)=3 H-E$ as in section 3 .

Put a Riemannian metric $g_{0}$ on $X$ so that the boundary $Y$ of a tubular neighbourhood of $\tilde{\Sigma}$ has a product metric. Arrange so that the metric on the factor $Y \cong S^{1} \times \Sigma$ is of the sort considered above. Let $X_{-}$and $X_{+}$ be respectively the closed tubular neighbourhood and the closure of its complement, and let $g_{R}$ be the Riemannian metric obtained by inserting a cylinder $[-R, R] \times Y$ between $X_{-}$and $X_{+}$.

Lemma 10. For $R$ sufficiently large, $c_{1}(L) \smile\left[\omega_{g_{R}}\right]$ is negative.

Proof. Let $R_{i}$ be a sequence increasing to infinity, and normalize the forms $\omega_{i}=\omega_{g_{R_{i}}}$ so that $H \smile\left[\omega_{i}\right]=1$. The forms $\omega_{i}$ then have $\left\|\omega_{i}\right\|_{L^{2}} \leq 1$ and there is a subsequence converging on compact sets to an $L^{2}$ form $\omega$ on the disjoint union of the two manifolds with cylindrical ends, $X_{-} \cup([0, \infty) \times Y)$ and $((-\infty, 0] \times Y) \cup X_{+}$. (The convergence is understood by identifying a compact subset of the cylindrical-end manifold with a compact subset of $\left(X_{R}, g_{R}\right)$ once $R$ is large enough.) However, the space of closed and coclosed $L^{2}$ 2-forms on $X_{-} \cup([0, \infty) \times Y)$ is empty, being the image of the compactly-supported cohomology in the ordinary cohomology. So $\left[\omega_{i}\right] \cdot[\tilde{\Sigma}]$ goes to zero as $i \rightarrow \infty$. Because of our normalization, we have

$$
\begin{aligned}
{\left[\omega_{i}\right] \smile c_{1}(L) } & =\left[\omega_{i}\right] \cdot[\tilde{\Sigma}]-(d-3)\left[\omega_{i}\right] \smile H \\
& =\left[\omega_{i}\right] \cdot[\tilde{\Sigma}]-(d-3) .
\end{aligned}
$$

Since $d$ is greater than 3, this quantity is eventually negative as claimed.

Corollary 7 now tells us that the moduli space $M_{c}\left(g_{R}\right)$ is non-empty for all sufficiently large $R$, and from Proposition 8 it follows that there is a solution on the cylinder $\mathbb{R} \times Y$ which is translation-invariant in a temporal gauge. Using Lemma 9 then, we obtain

$$
c_{1}(L)[\tilde{\Sigma}] \geq-(2 g-2)
$$

The left-hand side is $(3 H-E)(d H-E)$, which is $3 d-d^{2}$. This inequality is therefore equivalent to $2 g-2 \geq d^{2}-3 d$, which is equivalent to the inequality we wished to prove. 


\section{References}

1. S. K. Donaldson, Connections, cohomology and the intersection forms of fourmanifolds, J. Differential Geometry 24 (1986), 275-341.

2. __ Polynomial invariants for smooth four-manifolds, Topology 29 (1990), 257315.

3. M. Gromov and H. B. Lawson, The classification of simply connected manifolds of positive scalar curvature, Ann. of Math. 111 (1980), 423-434.

4. N. J. Hitchin, On the curvature of rational surfaces, In: Differential Geometry, Proc. Sympos. Pure Math., vol. 27, Amer. Math. Soc., Providence, R.I., 1975, pp. 65-80.

5. W. C. Hsiang and R. H. Szczarba, On embedding surfaces in four-manifolds, In: Algebraic Topology, Proc. Sympos. Pure Math., vol. 22, Amer. Math. Soc., Providence, R.I., 1971, pp. 97-103.

6. M. Kervaire and J. Milnor, On 2-spheres in 4-manifolds, Proc. Nat. Acad. Science USA 47 (1961), 1651-1657.

7. D. Kotschick and G. Matić, Embedded surfaces in four-manifolds, branched covers and SO(3)-invariants, Math. Proc. Camb. Phil. Soc. (to appear).

8. P. B. Kronheimer, The genus-minimizing property of algebraic curves, Bull. Amer. Math. Soc. 29 (1993), 63-69.

9. P. B. Kronheimer and T. S. Mrowka, Embedded surfaces and the structure of Donaldson's polynomial invariants, J. Differential Geometry (to appear).

10. V. A. Rochlin, Two-dimensional submanifolds of four-dimensional manifolds, Funct. Anal. Appl. 5 (1971), 39-48.

11. R. Schoen and S. T. Yau, On the structure of manifolds with positive scalar curvature, Manuscripta Math. 28 (1979), 159-183.

12. N. Seiberg and E. Witten, Electric-magnetic duality, monopole condensation, and confinement in $N=2$ supersymmetric Yang-Mills theory, preprint.

13. Monopoles, duality and chiral symmetry breaking in $N=2$ supersymmetric $Q C D$, preprint.

14. A. G. Tristram, Some cobordism invariants for links, Math. Proc. Camb. Phil. Soc. 66 (1969), 251-264.

15. E. Witten, Monopoles and four-manifolds, Math. Research Letters 1 (1994), 769796.

M athematical Institute, $24-29$ St. Giles, Oxford OX 13 L B

E-mail address: kronheim@maths.oxford.ac.uk

California Institute of Technology, Pasadena, CA 91125

E-mail address: mrowka@cco.caltech.edu 